telukast vor allem in der einmal tägliche Dosierung sowie in den fehlenden Nebenwirkungen. In der Studie war das Mittel genauso gut verträglich wie Plazebo. Das Mittel sei auch für Sportler geeignet. In den USA hätten viele Sportverbände sowie das Nationale Olympische Komitee bereite grünes Licht gegeben. Das Mittel hat gute Chancen, nicht als Doping klassifiziert zu werden.

Ein Nachteil sollte jedoch nicht verschwiegen werden. Er wurde auch in der Studie deutlich. Die Patienten sprechen sehr unterschiedlich auf das Medikament an. Während bei $23 \%$ der Patienten ein Abfall der FEV1 fast komplett verhindert wurde, erzielten $25 \%$ keine oder nur eine unwesentliche Protektion. Die Ärzte sollten den Patienten besser vor der Therapie sagen, daß die Chancen 1:4 stehen, daß das Medikament bei ihnen nicht wirkt. Einen Versuch sei es jedoch in jedem Fall wert.

(rme)

Quellen: Leff JA et al.: Montelukast, a leukotriene-receptor antagonist, for the treatment of mild asthma and exercise-induced bronchoconstriction. N Engl J Med 1998; 339: 147-52. Hansen-Flaschen J, Schotland H: New treatments for exercise-induced asthma. N Engl J Med 1998; 339: 192-3.

\section{Auch Salmeterol bessert die Lungenfunktion}

E ine weitere Alternative zur frühen Behandlung mit inhalativen Steroiden bei belastungsinduzierter Bronchokonstriktion ist Salmeterol. Dies zeigen die Ergebnisse einer randomisierten doppelblinden Crossover-Studie, welche Jo Ann Nelson, Universität Cleveland, an 20 Patienten mit belastungsinduziertem Asthma durchführte. Alle Patienten nahmen einen Monat lang zweimal täglich jeweils 2 Hübe Sal-

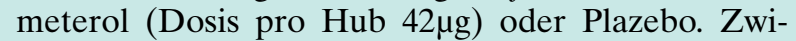
schen den beiden Behandlungen lag eine einwöchige Auswaschphase. Die Belastungstests mit dem Fahrradergometer (30 Minuten nach der Morgendosis) und 9 Stunden später zeigten, daß Salmeterol im Vergleich zu Plazebo das Ausmaß der Bronchokonstriktion zu allen Zeitpunkten abmildert. Die Fähigkeit des Arzneistoffs, seine Wirkung über den gesamten Tag auszuüben, ließ allerdings bei Langzeitverabreichung nach.

Quelle: Nelson JA et al.: Effect of long-term salmeterol treatment on exercice-induced asthma. N Engl J Med 1998; 339: 141-6.

\title{
Triamcinolon: gute Wirksamkeit bei allergischer Rhinitis
}

\author{
Zur Heuschnupfen-Behandlung wurde \\ in neues Kortisonpräparat synthetisiert, das sich durch \\ gute Wirksamkeit und Verträglichkeit auszeichnet.
}

(rme)

fü iamcinolon-Acetonid steht in wässriger Lösung als Nasenspray allergischen Rhinitis zur Verfug (als Dosieraerosol fuir die Behandlu des Asthma bronchiale befindet es sich Deutschland zur Zeit in der Ent-

Es konnte gezeigt werden, daß nasal appliziertes Triamcinolon nicht in signifikantem Ausmaß im Blutkreislauf eint und in therapeutischer Domiert: Im Gegensatz zu Prednison (10 $\mathrm{mg} / \mathrm{Tag})$ hatte Triamcinolon in wässriger Lösung (220 mg/Tag) während der 6wöchigen Behandlung keinen Einfluß auf die Nebennierenrindenfunktion.

Klinische Studien mit beiden Anwendungsformen haben gezeigt, daß das Präparat in einer Dosierung zwischen 110 und 220 $\mu \mathrm{g}$ einmal täglich verabreicht, die Heuschnupfensymptome bereits innerhalb der ersten Tage deutlich vermindert. Wenn die Symptome einmal unter Kontrolle sind, sollte die Dosierung reduziert werden (von 220 auf 110 $\mu \mathrm{g} / \mathrm{Tag})$, ohne daß ein Wirksamkeitsverlust zu befürchten wäre. Sowohl was die Symptomlinderung als auch den nasalen Eosinophilen-Influx betrifft, hat sich Triamcinolon gegenüber Plazebopräparaten als überlegen erwiesen.

Die Wirksamkeit einer einmal täglichen intranasalen Triamcinolon-Gabe (220 $\mu \mathrm{g} / \mathrm{Tag})$ war sowohl bezüglich subjektiver Symptome als auch bezüglich objektiver, mittels Rhinomanometrie erhobener Parameter den Vergleichspräparaten Beclomethason (84 bis $168 \mu \mathrm{g}$ zweimal täglich), Fluticason $(200 \mu \mathrm{g}$ einmal täglich) oder Flunisolid (100 $\mu \mathrm{g}$ zweimal täglich) über einen Zeitraum von 3 bis 12 Wochen ebenbürtig.

Im Vergleich zu den Antihistaminika Loratadin und Astemizol (jeweils oral eingenommen in einer täglichen Dosis von $10 \mathrm{mg}$ ) war Triamcinolon signifikant wirksamer - was die Linderung der nasalen Symptome angeht und genauso wirksam in der Linderung der begleitenden Augensymptomatik. Die Kombination von Triamcinolon mit oral verabreichtem Loratadin zeigte im Vergleich zur Monotherapie keine zusätzlichen Effekte.

In den bisher durchgeführten klinischen Untersuchungen wurde Triamcinolon-Acetonid gut vertragen. Einzige Nebenwirkungen waren Kopfschmerzen und Nasenbluten.

Insgesamt, so das Resümee der Autoren, kann Triamcinolon als Nasenspray aufgrund der vorliegenden Daten als Therapeutikum bei Erwachsenen mit mäßig schwerer saisonaler allergischer Rhinitis, aber auch bei Kindern und Erwachsenen mit perennialer allergischer Rhinitis empfohlen werden.

(mo)

Quelle: Jeal W et al.: Triamcinolone Acetonide. A Review of its Pharmacological Properties and Therapeutic Efficacy in 